\section{Kommt 2016 der Durchbruch?}

\author{
Für die Allgemeinmedizin bricht ein Schicksalsjahr an: Ein Masterplan \\ der Regierung zum Medizinstudium und neue Kompetenzzentren für \\ die Weiterbildung könnten Bewegung in die Nachwuchsfrage bringen.
}

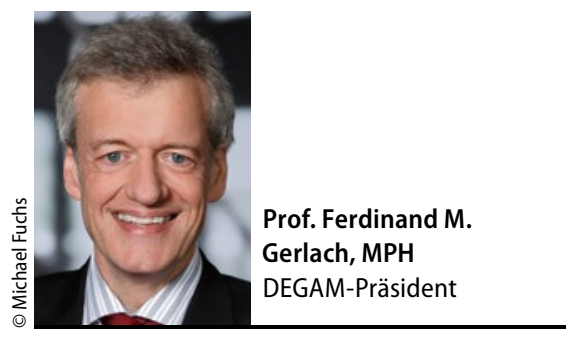

_ Aktuell findet nur jeder zweite Hausarzt einen Nachfolger. Wir müssten mindestens doppelt so viele Fachärzte für Allgemeinmedizin weiterbilden, wie dies derzeit der Fall ist. Die gute Nacharzt für Allgemeinmedizin ist zuletzt leicht gestiegen. Wenn wir zielgerichtete Maßnahmen umsetzen, ist auch eine Verdopplung der Zahlen absolut realistisch!

Im 2014 durchgeführten Berufsmonitoring kam für $34,5 \%$ der mehr als 11.000 bundesweit befragten Medizinstudierenden eine Weiterbildung im Fach Allgemeinmedizin in Frage. 2010 konnten sich das erst 29,3\% vorstellen: ein absoluter Zuwachs von 5,2\% und Platz 2 unter allen Fächern. Es geht also „nur“ noch darum, dieses Potenzial auch auszuschöpfen. Für das Gelingen sind neben den Rahmenbedingungen der späteren Praxistätigkeit - zwei Dinge zentral: die Rolle der Allgemeinmedizin in der universitären Ausbildung sowie die Art und Qualität der Weiterbildung zukünftiger Fachärzte für Allgemeinmedizin.

In den nächsten Monaten soll in einer gemeinsamen Konferenz der Gesundheits- und Wissenschaftsminister des Bundes und der Länder ein „Masterplan Medizinstudium 2020“ verabschiedet werden. Eines von drei Zielen ist die Stärkung der Allgemeinmedizin im Studium. Gelingt es, Allgemeinmedizin zu einem obligatorischen Bestandteil des Praktischen Jahres und der abschließenden mündlich-praktischen M3-Prüfung zu machen, wäre ein wichtiger Durchbruch erreicht. Die Stärkung des Fachs an diesen Schlüsselpositionen würde der richt: Die Zahl der Abschlüsse als Fach-
Rolle der Allgemeinmedizin in der Versorgung gerecht, $\mathrm{zu}$ mehr Breite und Qualität im Medizinstudium beitragen und mehr Studierende zu einer allgemeinmedizinischen Weiterbildung motivieren.

\section{Angehende Hausärzte sollen auch emotional gestärkt werden}

Eine zweite wesentliche Entscheidung wird ebenfalls in den nächsten Monaten fallen: Die Rahmenvereinbarung zur Förderung der Weiterbildung nach $\$ 75 \mathrm{a}$ SGB V wird überarbeitet. Das betrifft vor allem die Weiterbildung Allgemeinmedizin in Kliniken und Praxen. Neu und für den Gesamterfolg ausschlaggebend ist die erstmals mögliche bundesweite Förderung von universitär angebundenen Kompetenzzentren, die es bisher nur in Heidelberg, Frankfurt und Marburg gibt. Hier erhalten junge Ärzte in Weiterbildung die notwendige emotionale Rückenstärkung und fachliche Unterstützung. Die Kompetenzzentren errei- chen dies durch ein Mentoring, welches auf bestehende Sorgen und Ängste eingeht, sowie durch Begleitseminare, die auf fachliche und organisatorische Herausforderungen der Praxistätigkeit vorbereiten. Statt sich einsam durchzuwursteln, findet der allgemeinmedizinische Nachwuchs hier eine emotionale und fachliche Heimat. Folge: Der Nachwuchs ist erleichtert und findet Allgemeinmedizin, spezialisiert auf den ganzen Menschen, sogar cool.

Gelingt es, diese wegweisenden Verbesserungen in der Aus- und Weiterbildung des dringend benötigten Nachwuchses umzusetzen, käme das einem Durchbruch in der Nachwuchsfrage gleich. Die Allgemeinmedizin bekäme Flügel - und die Chancen, dass auch zukünftig eine flächendeckende gesundheitliche Versorgung der Bevölkerung sichergestellt werden kann, würden sich dramatisch verbessern.

- Der Autor ist Präsident der Deutschen Gesellschaft für Allgemeinmedizin und Familienmedizin (DEGAM)

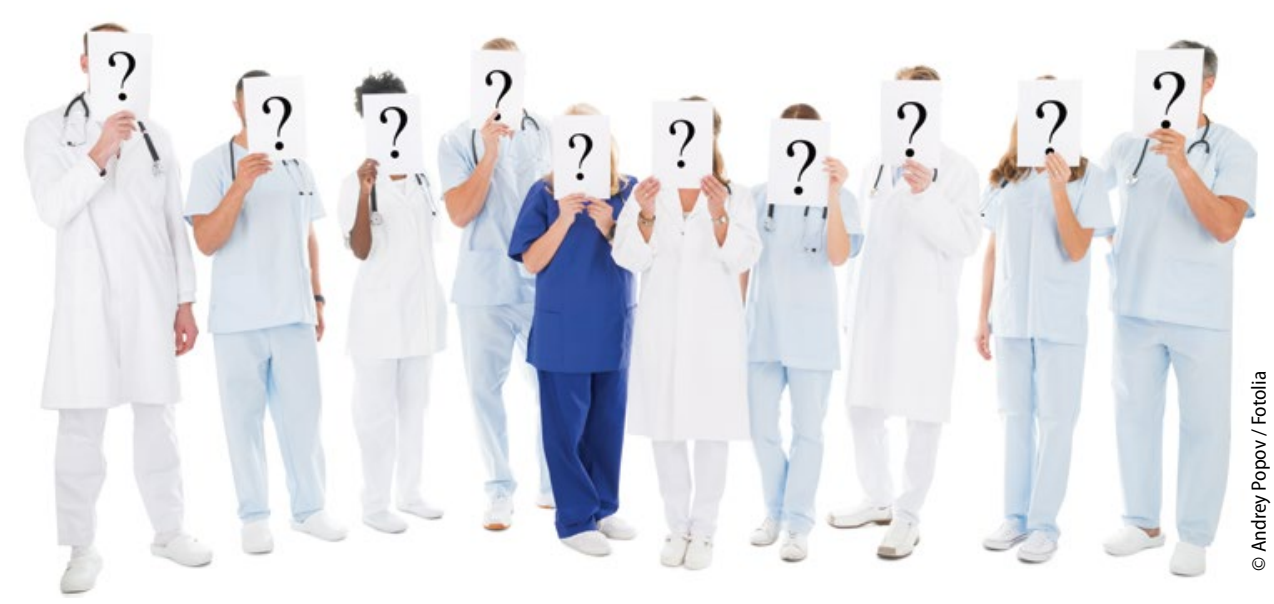

Wer von ihnen wird Hausarzt? Möglichst viele, wie wir hoffen! 